\title{
Mid-term follow-up surgical results in 284 cases of clival chordomas: the risk factors for outcome and tumor recurrence
}

\author{
Jiwei Bai ${ }^{1,2,3} \cdot$ Mingxuan $\mathrm{Li}^{1,3}$. Jianxin Shi ${ }^{4}$. Liwei Jing ${ }^{5}$ Yixuan Zhai ${ }^{6}$. Shuheng Zhang ${ }^{7}$. Junmei Wang ${ }^{1}$. \\ Peng Zhao ${ }^{2}$. Chuzhong $\mathrm{Li}^{1}$ · Songbai Gui ${ }^{2}$. Yazhuo Zhang ${ }^{1,2,3}$
}

Received: 15 March 2021 / Revised: 12 May 2021 / Accepted: 26 May 2021 / Published online: 8 October 2021

(c) The Author(s) 2021

\begin{abstract}
Objective Skull base chordoma (SBC) is rare and one of the most challenging diseases to treat. We aimed to assess the optimal timing of adjuvant radiation therapy (RT) and to evaluate the factors that influence resection and long-term outcomes. Methods In total, 284 patients with 382 surgeries were enrolled in this retrospective study. Postsurgically, 64 patients underwent RT before recurrence (pre-recurrence RT), and 47 patients underwent RT after recurrence. During the first attempt to achieve gross-total resection (GTR), when the entire tumor was resected, 268 patients were treated with an endoscopic midline approach, and 16 patients were treated with microscopic lateral approaches. Factors associated with the success of GTR were identified using $\chi^{2}$ and logistic regression analyses. Risk factors associated with chordoma-specific survival (CSS) and progression-free survival (PFS) were evaluated with the Cox proportional hazards model.

Results In total, $74.6 \%$ of tumors were marginally resected [GTR (40.1\%), near-total resection (34.5\%)]. History of surgery, large tumor volumes, and tumor locations in the lower clivus were associated with a lower GTR rate. The mean follow-up period was 43.9 months. At the last follow-up, 181 (63.7\%) patients were alive. RT history, histologic subtype (dedifferentiated and sarcomatoid), non-GTR, no postsurgical RT, and the presence of metastasis were associated with poorer CSS. Patients with pre-recurrence RT had the longest PFS and CSS, while patients without postsurgical RT had the worst outcome. Conclusion GTR is the goal of initial surgical treatment. Pre-recurrence RT would improve outcome regardless of GTR.
\end{abstract}

Keywords Chordoma $\cdot$ Prognosis $\cdot$ Radiation therapy $\cdot$ Skull base $\cdot$ Surgical approaches

Yazhuo Zhang

zyz2004520@163.com

1 Beijing Neurosurgical Institute, Capital Medical University, Beijing, China

2 Department of Neurosurgery, Beijing Tiantan Hospital, Capital Medical University, Beijing, China

3 China National Clinical Research Center for Neurological Diseases, Beijing, China

4 Division of Cancer Epidemiology and Genetics, National Cancer Institute, NIH, DHHS, Bethesda, MD, USA

5 Department of Health Statistics, Shanxi Medical University, Taiyuan, China

6 Department of Neurosurgery, First Affiliated Hospital, Zhengzhou University, Zhengzhou, China

7 Department of Neurosurgery, Anshan Central Hospital, Anshan, China

\section{Introduction}

Chordoma is a rare bone malignancy with an incidence rate of 0.08 and 0.04 per 100,000 in the USA and Europe [11, 20] and in Taiwan [13], respectively. Skull base chordomas (SBCs) account for $32 \%$ of all chordomas [20]. Local recurrence is common with a late recurrence rate $>50 \%$ [8, $10,14,25,30]$. The average survival after surgery with or without radiation therapy (RT) is approximately 7.7 years [35]. Because of the deep location and close proximity to vital structures, surgical treatment is a challenge for SBCs with the total resection rate ranging from 0 to $73.7 \%$ [8]. The endoscopic midline approach (EMA) for SBCs yields better or similar resections than the microscopic lateral open approach (MLOA) [12, 26]. Factors that influence resection with EMA still need to be clarified.

Although RT is recommended as an adjuvant treatment to surgery [24], the use of RT remains controversial [7, 8, 23, 40]. In clinical practice, it is not clear whether and when RT 
should be administered [23,31], especially when the tumor was gross-total resected [33]. Hence, a comprehensive investigation of the optimum management protocol conducted in a large study is warranted $[8,14]$.

Our group, the first neuroendoscopic group in China, was established in Beijing Tiantan Hospital in 1998 and has since then focused on skull base diseases [18]. We have used both EMA and MLOA to resect SBCs during the past two decades. In this study, we retrospectively analyzed the clinical data of 284 patients with SBCs who were treated by our single group to identify factors for achieving complete resection and the optimal timing for RT.

\section{Methods}

\section{Patients}

Patients with histologically confirmed SBCs who were treated between December 31, 2003, and January 31, 2019, were included in the present study. The patients' clinical data and follow-up information were retrospectively reviewed. This study was approved by the ethics committee of Beijing Tiantan Hospital (KY2018-053-02). All patients signed informed consent forms for surgery treatment preoperatively. Because of the retrospective nature of the present study and that the results will not affect the treatment strategy for patients, no additional informed consent was required to participate in the study.

\section{Radiological evaluation}

All patients underwent MRI and CT examinations pre-operatively. The MRI sequences mainly include T1-weighted images in the sagittal and axial planes, T2-weighted images in the axial plane, and postcontrast T1-weighted images in the sagittal, axial, and coronal planes. The sequence parameters were as follows: T1WI (axial), FOV $240 \times 240,24$ slices, $\mathrm{TE}=28.16 \mathrm{~ms}, \mathrm{TR}=2275 \mathrm{~ms}$, and TI $($ inversion time) $=950 \mathrm{~ms}$; T2WI, FOV $240 \times 240$, 24 slices, $\mathrm{TE}=147 \mathrm{~ms}, \mathrm{TR}=8000 \mathrm{~ms}$, and TI (inversion time $)=2000 \mathrm{~ms}$; and DWI, FOV $240 \times 240,24$ slices, $\mathrm{TE}=63.2 \mathrm{~ms}$, and $\mathrm{TR}=2300 \mathrm{~ms}$. Each patients' pre- and post-operative images were evaluated by both radiologist on duty and our neuroendoscopic team. The tumor volume was calculated as volume $=(\mathrm{a} \times \mathrm{b} \times \mathrm{c}) / 2$, where $\mathrm{a}, \mathrm{b}$, and $\mathrm{c}$ represent the longest diameters in sagittal, coronal, and axial views, respectively. According to the sellar floor and sphenoid sinus floor plane in sagittal view, the clivus was divided into the superior, middle, and inferior zones, as previously described by our group (Fig. 1A) [12]. According to the bilateral boundaries traditionally established for EMA, the skull base was divided into midline and paramedian regions (Fig. 1B) [12]. When $>1 / 2$ circumference of the internal carotid artery (ICA) was encased, cavernous sinus invasion was defined. Tumors that had invaded into the subdural space were defined as exhibiting dural penetration [36].

\section{Surgical methods}

We used both EMAs (transnasal or transoral or combined) and MLOAs, which were chosen according to the tumor location [12]. When a tumor was located in the midline region or was slightly extended to the paramedian region, EMA was the first choice. When the tumor was mainly located in the paramedian region, we chose MLOA. Based on our belief that the dura is a natural barrier against intracranial invasion by chordomas, we did not cut off the inner
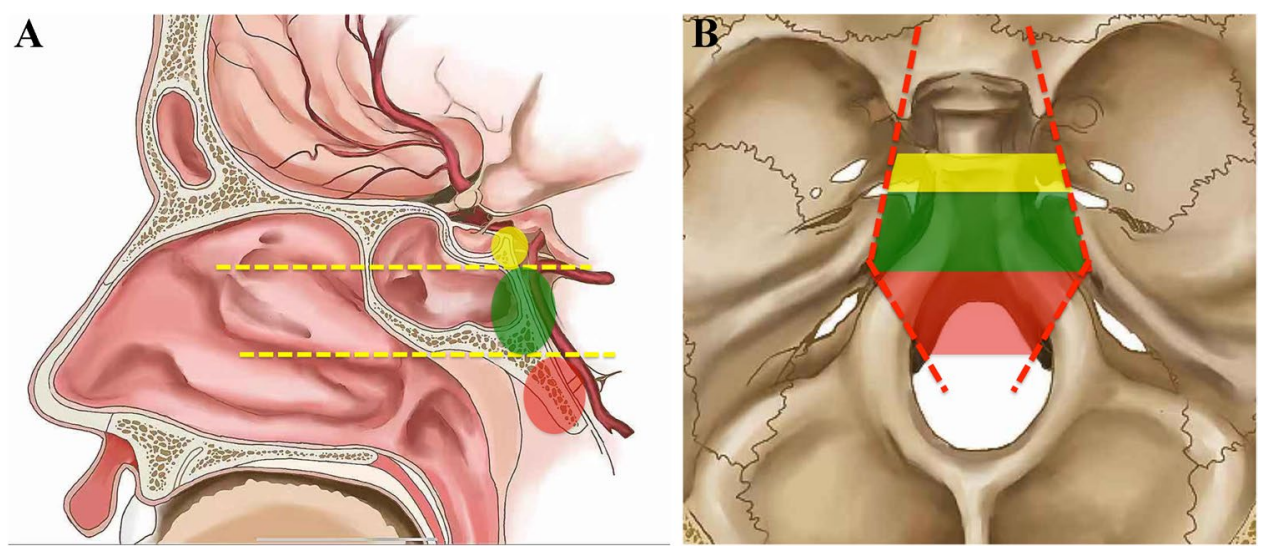

Fig. 1 Anatomic classification of the clival region. A Sagittal view, the upper yellow horizontal line represents the sellar floor plane; the lower yellow horizontal line represents the sphenoid sinus floor plane. The yellow part represents the superior clivus; the green part represents the middle clivus; and the red part represents the inferior clivus.
B Axial view, the red lines represent the bilateral lines connecting the lateral wall of the cavernous sinus, internal auditory canal, hypoglossal nerve hole, and occipital condyle. The region between the red line is the midline region of the skull base, and the region beyond the red line is the paramedian region 
layer of dura but thoroughly resected the tumor tissue that attached to the dura in patients with primary chordomas. When dural penetration existed [36], we followed the tumor passage and resected it. For patients with primary chordomas, a typical multi-layer reconstruction of clival defects was consisted of inlay collagen substitutes and autologous fat pad, onlay autologous and fascia lata, and vascularized nasoseptal flap. For patients with recurrent chordomas whose nasoseptal flap was not available, repairing the defects with absorbable collagen substitutes and autologous fascia lata was usually effective to prevent CSF leakage. In a few complicated cases, such as the patients have RT history and the defects were larger, we would deliberately preserve the most inner layer of chordoma in order to avoid high-flow CSF leak, which is a balance between the risk of repairing failure and the quality of life. When the tumor volume that invaded into subdural space was large and the dural defect after tumor removal was large and no vascularized flap was available, we will suture the autologous fat pad to normal dural edge using 5-0 prolene stitches besides the above-mentioned multi-layer reconstruction method. If refractory CSF happened, pedicle temporalis muscle flap was extremely useful. Post-operative lumbar drainage was generally placed for 5 days for patients with large defects. The resection rate was divided into four grades according to the postsurgical MRI and CT combined with the intraoperative impressions of surgeons [30]. CT images were scanned on the night of surgery day, and MR images were acquired within 2 days after operation except severe complications happen. Gross-total resection (GTR) indicated that the entire tumor was resected, the surrounding healthy tissue was obviously exposed, and no suspected tumor could be found on postsurgical images. No residual tumor was found during surgery, and $>90 \%$ tumoral resection on images was defined as near-total resection (NTR); $>70 \%$ tumoral resection on images was defined as subtotal resection (STR), and a lesser extent of resection was defined as partial resection (PR). For the sake of comparison, GTR and NTR were pooled and classified as marginal resection, and STR and PR were classified as intralesional resection $[24,25,30]$.

\section{Follow-up}

The first follow-up was performed in outpatient center after surgery. Thereafter, enhanced MRI was done every 6 months. RT was recommended to all patients. The date of the last follow-up, which was conducted by telephone, was November 9, 2019. Inquiries were made regarding RT modalities and dates. RT modalities include radiosurgery (Gamma Knife and Cyberknife), intensity-modulated photon radiotherapy (IMRT), and charged particle radiotherapy (CPRT, which includes both proton and carbon ion RT). Tumor recurrence was confirmed by radiological imaging.
Progression-free survival (PFS) was calculated from the date of surgery to the date of radiographic recurrence. Chordomaspecific survival (CSS) was defined as the time between the date of surgery and the date of death caused by chordoma. If a patient was lost to follow-up or died of surgical complications or non-disease-specific reasons, censor data were applied.

\section{Statistical analyses}

Statistical analyses were performed using SPSS 19 (IBM Corp, Armonk, New York) or R version 3.6.1 (R Foundation for Statistical Computing, Vienna, Austria). The difference in age distributions between groups was tested using the Mann-Whitney U test. PFS and CSS were estimated with the Kaplan-Meier method. To evaluate the influence of RT timing on CSS, patients were classified into three categories: RT before recurrence (pre-recurrence RT), RT after recurrence (late RT), and no RT. A univariate Cox proportional hazards model was performed to evaluate the marginal association between a potential prognostic factor and CSS/PFS. For factors with $P$ value $\leq 0.05$ in marginal analyses, we performed multivariate Cox proportional hazards regression to jointly estimate the effect of these factors. We used $\chi^{2}$ test to evaluate the association between factors and the resection rate; factors with $P \leq 0.05$ were then jointly analyzed using logistic regression. Factors with $P<0.05$ in joint analyses were considered statistically significant.

\section{Results}

\section{Patients' demographic and clinical characteristics}

A total of 284 patients underwent 382 surgeries (Table 1), including 380 skull base surgeries (349 EMAs and 31 MLOAs) and two metastatic lesion resections. Common presenting symptoms included diplopia (50.7\%), headache or neck pain (34.5\%), and blurry vision (24.6\%). The median duration from self-reported initial symptoms to diagnosis was 6.0 months (range 1.0-108.0 months). A total of 184 patients were newly diagnosed, and 100 patients had a treatment history. Marginal resection was achieved for $74.6 \%$ of patients, including $114(40.1 \%)$ undergoing GTR and 98 (34.5\%) undergoing NTR. Intralesional resection was achieved in $25.4 \%$ of tumors, including 64 (22.5\%) with STR and eight $(2.8 \%)$ with PR. A total of 111 patients underwent postsurgical RT, including 64 patients with pre-recurrence RT (mean time between surgery and RT, 3.5 months; range, 1-13 months) and 47 patients with late RT (mean time, 21.7 months; range, 4-79 months). 
Table 1 Demographic and clinical characteristics of 284 patients with clivus chordomas

\begin{tabular}{|c|c|c|}
\hline & & No. $(\%)$ \\
\hline \multirow[t]{2}{*}{ Sex } & Male & $162(57.0 \%)$ \\
\hline & Female & $122(43.0 \%)$ \\
\hline \multirow[t]{2}{*}{ Tumor status } & Primary & $184(64.8 \%)$ \\
\hline & Recurrent & $100(35.2 \%)$ \\
\hline \multirow[t]{5}{*}{ Age, years ${ }^{a}$} & Median (range) & $44.0(3.0-77.0)$ \\
\hline & Male & $44.0(5.0-76.0)$ \\
\hline & Female & $43.5(3.0-77.0)$ \\
\hline & Primary & $44.0(3.0-77.0)$ \\
\hline & Recurrent & $43.5(3.5-70.0)$ \\
\hline \multirow[t]{2}{*}{ RT history } & Yes & $47^{\mathrm{b}}(16.5 \%)$ \\
\hline & No & $237(83.5 \%)$ \\
\hline \multirow[t]{3}{*}{ Previous RT modality } & Radiosurgery & $41(87.2 \%)$ \\
\hline & IMRT & $2(4.3 \%)$ \\
\hline & CPRT & $4(8.5 \%)$ \\
\hline \multirow[t]{4}{*}{ Postsurgery RT } & Yes (pre-recurrence RT) & $64(22.5 \%)$ \\
\hline & Yes (late RT) & $47(16.5 \%)$ \\
\hline & No & $147(51.8 \%)$ \\
\hline & NA & $26(9.2 \%)$ \\
\hline \multirow[t]{4}{*}{ Postsurgery RT modality } & Radiosurgery & $40(36.0 \%)$ \\
\hline & IMRT & $33(29.7 \%)$ \\
\hline & CPRT & $31(27.9 \%)$ \\
\hline & NA & $7(6.3 \%)$ \\
\hline Tumor volume median (range) $\left(\mathrm{cm}^{3}\right)$ & & $22.3(0.91-258.0)$ \\
\hline \multirow[t]{3}{*}{ Dural penetration } & Yes & $138(48.6 \%)$ \\
\hline & No & $136(47.9 \%)$ \\
\hline & Unclear $^{c}$ & $10(3.5 \%)$ \\
\hline \multirow[t]{3}{*}{ Histologic subtype } & Conventional & $216(76.1 \%)$ \\
\hline & Chondroid & $63(22.2 \%)$ \\
\hline & Dedifferentiated ${ }^{\mathrm{d}}$ & $5(1.8 \%)$ \\
\hline \multirow[t]{3}{*}{ Metastasis } & Yes & $9(3.2 \%)$ \\
\hline & No & $242(85.2 \%)$ \\
\hline & NA & $33(11.6 \%)$ \\
\hline
\end{tabular}

a No significant difference between sexes $(P=0.65)$ or between primary and recurrent tumors $(P=0.61)$.

${ }^{b}$ Including 17 patients with radiotherapy as initial treatment, and 30 patients with recurrent tumor who have underwent surgeries and followed by RT treatment previously. ${ }^{c}$ Unclear, difficult to judge by radiologic evaluation and surgical records. ${ }^{\mathrm{d}}$ Included four dedifferentiated chordomas and one sarcomatoid chordoma. $R T$, radiation therapy. $N A$, not available. IMRT, intensity-modulated photon radiotherapy. $C P R T$, charged particle radiotherapy

\section{Factors associated with CSS}

The average follow-up time was 43.9 months (median 32.5, range 2-175). At the last follow-up, 181 patients were alive. The 5-year CSS was $71.0 \%$ (95\% CI $=62.8-80.2 \%$ ). The patients with marginal resection had significantly longer survival times than those with intralesional resection $\left(\mathrm{HR}=0.18,95 \% \mathrm{CI}=0.089-0.360, P=1.5 \times 10^{-6}\right)$. We performed a more detailed analysis and found that GTR significantly improved survival, while other groups (i.e., NTR, STR, and PR) did not show significant differences (Fig. 2A).
We found no significant difference in CSS between conventional and chondroid chordomas ( $\mathrm{HR}=1.57,95 \%$ $\mathrm{CI}=0.70-3.49, P=0.273)$. For clarity, we combined conventional and chondroid chordomas into one group and classified the resection rate into GTR and non-GTR. The results based on univariate analysis are reported in Supplementary Table 1. The 5-year CSS was higher in the GTR group $[90.3 \%(95 \% \mathrm{CI}=83.6-97.6 \%)]$ than in the non-GTR group [49.8\% (95\% CI=38.8-63.8\%)] (Fig. 2B). The group with pre-recurrence RT had significantly longer CSS than both the group without RT and the group with late RT (Fig. 2C). Based on the multivariate Cox proportional hazards model, 


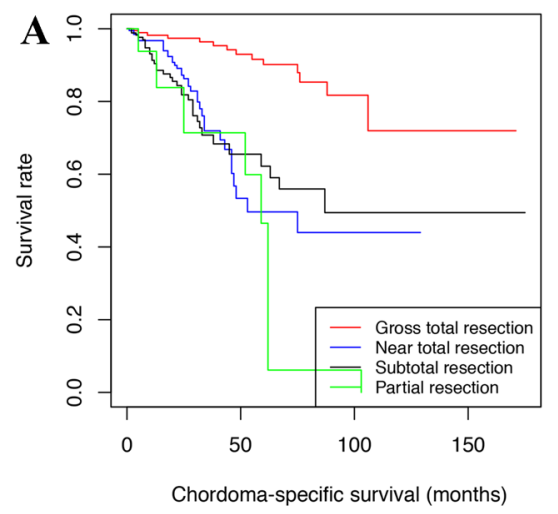

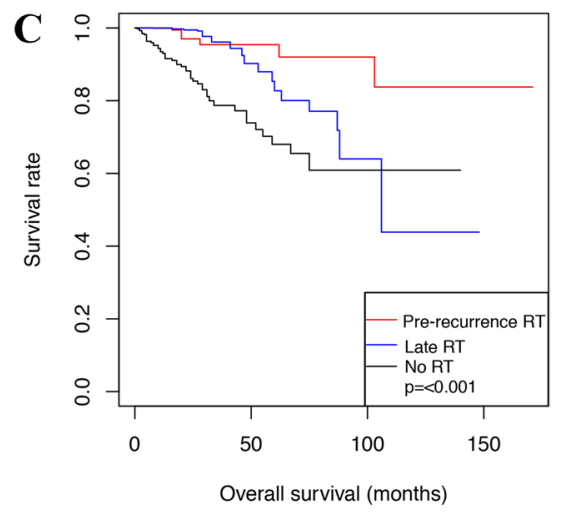

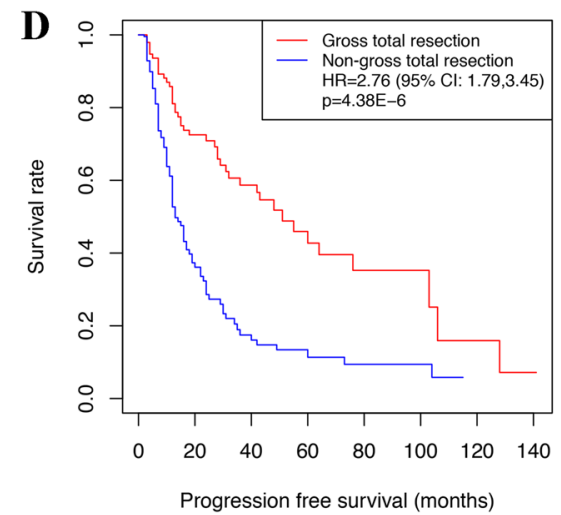

Fig. 2 Risk factors for chordoma-specific survival (CSS) and progression-free survival (PFS) in all 284 patients. A The CSS was significantly longer in the gross-total resection (GTR) group than in the near-total resection (NTR) group, subtotal resection (STR) group, or partial resection $(\mathrm{PR})$ group $(P<.05)$. B When the NTR group, STR group, and PR group were classified into the non-gross-total resection group (non-GTR), the CSS was longer in the GTR group than in the

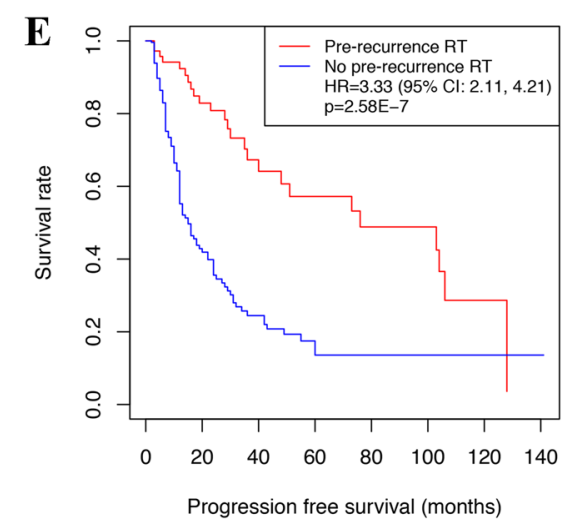

non-GTR group $(P<.05)$. C The group with pre-recurrence radiation therapy (RT) had significantly longer CSS than both the group without RT and the group with late RT $(P<.05)$. D PFS was longer in the GTR group than in the non-GTR group $(P<.05)$. $\mathbf{E}$ The patients who underwent RT before recurrence (pre-recurrence RT) had a longer PFS than the other patients (no pre-recurrence RT) $(P<.05)$

on the multivariate Cox proportional hazards model, we found that non-GTR $(\mathrm{HR}=2.76,95 \% \mathrm{CI}=1.79-3.45$, $P<0.001$ ) (Fig. 2D), no pre-recurrence RT (HR $=3.33$, 95\% CI $=2.11-4.21, P<0.001$ ) (Fig. 2E), history of RT $(\mathrm{HR}=1.80,95 \% \mathrm{CI}=1.05-2.37, P=0.033)$, and history of surgery $(\mathrm{HR}=1.59,95 \% \mathrm{CI}=1.04-1.97, P=0.034)$ were associated with shorter PFS (Table 3).

\section{Factors associated with long-term outcomes in primary SBCs}

To further clarify the effect of risk factors for CSS and PFS, we restricted analyses to 184 patients with primary tumors. The results showed that non-GTR $(\mathrm{HR}=2.48$, 95\% CI $=1.45-4.23, P<0.001$ ) (Fig. 3A), tumor location in the lower $2 / 3$ of the clivus (HR $=2.54,95 \%$ $\mathrm{CI}=1.32-4.87, P=0.005)$, cavernous invasion $(\mathrm{HR}=1.96$, $95 \% \mathrm{CI}=1.03-3.70, P=0.041)$, late $\mathrm{RT}(\mathrm{HR}=2.77,95 \%$ $\mathrm{CI}=1.43-5.35, P=0.002)$, and no RT $(\mathrm{HR}=1.98,95 \%$ $\mathrm{CI}=1.09-3.60, P=0.026)$ were statistically associated 
Table 2 Factors associated with chordoma-specific survival in patients with skull base chordomas. Analysis was based on a multivariate Cox proportional hazards model

\begin{tabular}{|c|c|c|c|c|}
\hline \multicolumn{2}{|l|}{ Variable } & \multirow{2}{*}{$\begin{array}{l}\text { Hazard ratio } \\
1.65\end{array}$} & \multirow{2}{*}{$\frac{95 \% \mathrm{CI}}{1.02-2.68}$} & \multirow{2}{*}{$\frac{P \text { value }}{.043}$} \\
\hline Sex & Female vs. male & & & \\
\hline \multirow[t]{2}{*}{$\operatorname{Age}^{a}$} & Group 2 vs. group 1 & 1.77 & $0.79-3.95$ & .162 \\
\hline & Group 3 vs. group 1 & 1.67 & $0.62-4.53$ & .314 \\
\hline Histopathologic subtype & Dedifferentiated vs. conventional/chondroid & 8.21 & $2.27-29.67$ & .001 \\
\hline History of surgery & Yes vs. no & 1.45 & $0.75-2.79$ & .273 \\
\hline History of RT & Yes vs. no & 2.04 & $1.06-3.93$ & .034 \\
\hline Resection rate & Non-GTR vs. GTR & 4.71 & $2.28-9.76$ & $<.001$ \\
\hline \multirow[t]{3}{*}{ Tumor location (Sagittal view) } & Lower $2 / 3$ vs. upper $2 / 3$ & 2.32 & $1.15-4.67$ & .018 \\
\hline & Total clivus vs. upper $2 / 3$ & 1.17 & $0.64-2.14$ & .603 \\
\hline & Others vs. upper $2 / 3$ & 0.38 & $0.08-1.72$ & .207 \\
\hline Tumor location (Axial view) ${ }^{b}$ & $\begin{array}{l}\text { Median extension to the paramedian region vs. the } \\
\text { midline }\end{array}$ & 0.70 & $0.40-1.21$ & .197 \\
\hline Cavernous sinus invasion & Yes vs. no & 2.16 & $1.16-4.05$ & .016 \\
\hline Dural penetration & Yes vs. no & 1.35 & $0.79-2.32$ & .274 \\
\hline Tumor volume & $\leq 40$ vs. $>40$ & 0.67 & $0.38-1.21$ & .187 \\
\hline \multirow[t]{2}{*}{ Postsurgical RT } & Late RT vs. pre-recurrence RT & 2.02 & $0.76-5.36$ & .160 \\
\hline & No RT vs. pre-recurrence RT & 4.87 & $2.03-11.65$ & $<.001$ \\
\hline Metastasis & Yes vs. no & 5.39 & $1.96-14.84$ & .001 \\
\hline
\end{tabular}

${ }^{a}$ Age was classified into three groups, e.g., group 1 represented $\leq 20$ years of age, group 2 represented between 20 and 60 years of age, and group $\mathrm{C}$ represented $\geq 60$ years of age. ${ }^{\mathrm{b}}$ One patient whose tumor was located in the paramedian region was included in the group with median extension to the paramedian region

The bolded entries are significant $p$-values

with poor CSS. Moreover, non-GTR (HR $=3.20$, 95\% $\mathrm{CI}=1.91-5.37, P<0.001)($ Fig. $3 \mathrm{~B})$ and no pre-recurrence $\mathrm{RT}(\mathrm{HR}=2.70,95 \% \mathrm{CI}=1.56-4.67, P<0.001)($ Fig. $3 \mathrm{C})$ significantly increased the risk of recurrence.

\section{Factors associated with the failure of GTR}

Given the importance of GTR, we performed a multivariate logistic regression analysis to identify factors that are
Table 3 Factors associated with progression-free survival in patients with skull base chordomas. Analysis was based on a multivariate Cox proportional hazards model

\begin{tabular}{|c|c|c|c|c|}
\hline \multicolumn{2}{|l|}{ Variable } & \multirow{2}{*}{$\begin{array}{l}\text { Hazard ratio } \\
0.99\end{array}$} & \multirow{2}{*}{$\frac{95 \% \mathrm{CI}}{0.69-1.20}$} & \multirow{2}{*}{$\begin{array}{l}P \\
.971\end{array}$} \\
\hline Sex & Female vs. male & & & \\
\hline \multirow[t]{2}{*}{$\operatorname{Age}^{a}$} & Group 2 vs. group 1 & 1.53 & $0.93-1.98$ & .097 \\
\hline & Group 3 vs. group 1 & 1.44 & $0.75-2.01$ & .274 \\
\hline Histopathologic subtype & $\begin{array}{l}\text { Dedifferentiated vs. conven- } \\
\text { tional/chondroid }\end{array}$ & 2.40 & $0.65-4.65$ & .188 \\
\hline History of surgery & Yes vs. no & 1.59 & $1.04-1.97$ & .034 \\
\hline History of RT & Yes vs. no & 1.80 & $1.05-2.37$ & .033 \\
\hline Resection rate & Non-GTR vs. GTR & 2.76 & $1.79-3.45$ & $<.001$ \\
\hline \multirow[t]{3}{*}{ Tumor location (sagittal view) } & Lower $2 / 3$ vs. upper $2 / 3$ & 1.05 & $0.65-1.35$ & .836 \\
\hline & Total clivus vs. upper $2 / 3$ & 0.93 & $0.59-1.18$ & .760 \\
\hline & Others vs. upper $2 / 3$ & 0.53 & $0.20-0.86$ & .193 \\
\hline Tumor location (axial view) ${ }^{b}$ & Paramedian vs. midline & 1.23 & $0.83-1.51$ & .295 \\
\hline Cavernous sinus invasion & Yes vs. no & 1.13 & $0.73-1.41$ & .588 \\
\hline Dural penetration & Yes vs. no & 0.85 & $0.59-1.02$ & .378 \\
\hline Tumor volume & $>40 \mathrm{vs} . \leq 40 \mathrm{~cm}^{3}$ & 1.03 & $0.67-1.28$ & .889 \\
\hline Postsurgical RT & No RT vs. RT & 3.33 & $2.11-4.21$ & $<.001$ \\
\hline
\end{tabular}

a Age was classified into three groups, e.g., group 1 represented $\leq 20$ years of age, group 2 represented between 20 and 60 years of age, and group 3 represented $\geq 60$ years of age. ${ }^{b}$ One patient whose tumor was located in the paramedian region was included in the group with median extension to the paramedian region

The bolded entries are significant $p$-values 
Fig. 3 Risk factors for chordoma-specific survival (CSS) and tumor recurrence in 184 primary chordoma patients. A Non-gross-total resection (nonGTR) was statistically associated with poorer CSS $(P<.001)$. B Non-GTR $(P<.001)$ and $\mathbf{C}$ no pre-recurrence $\mathrm{RT}(P<.001)$ significantly increased the risk of recurrence
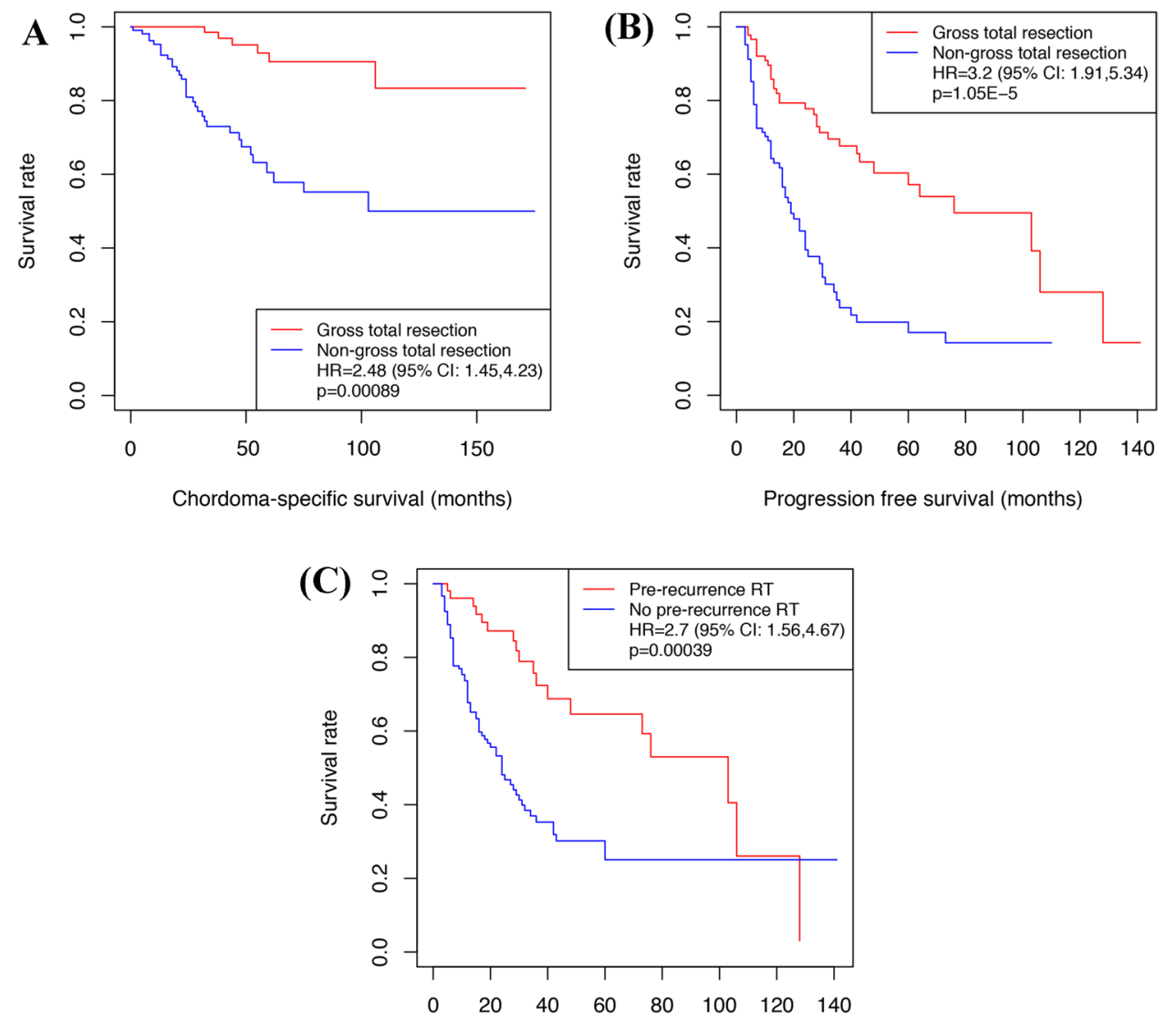

Progression free survival (months)

associated with the failure of GTR. We found that younger age $(\mathrm{OR}=0.28,95 \% \mathrm{CI}=0.09-0.87, P=0.028)$, history of surgery $(\mathrm{OR}=3.45,95 \% \mathrm{CI}=1.59-7.49, P=0.002)$, tumor location (lower $2 / 3$ of the clivus, $\mathrm{OR}=8.73,95 \%$ $\mathrm{CI}=3.12-24.45, P<0.001$; total clivus, $\mathrm{OR}=3.07,95 \%$ $\mathrm{CI}=1.36-6.94, P=0.007$; invasion into the paramedian region, $\mathrm{OR}=2.88,95 \% \mathrm{CI}=1.42-5.82, P=0.003)$, and large tumor volume $\left(>40 \mathrm{~cm}^{3}\right)(\mathrm{OR}=3.07,95 \%$ $\mathrm{CI}=1.22-7.76, P=0.018)$ were associated with the failure of GTR (Table 4) (see also Supplementary Table 2 for univariate logistic regression analyses). The GTR rate showed no significant difference between different approaches (EMA 40.3\% and MLOA 37.5\%, $P=0.82$ ) (Supplementary Table 2).

\section{Surgical complications}

Complications were comprised of the following: cranial nerve injury (7.9\%), cerebrospinal fluid (CSF) leakage $(3.9 \%)$, intracranial infection $(3.2 \%)$, hypopituitarism (2.1\%), severe pneumonia followed by lower cranial injury $(1.6 \%)$, postsurgical hematoma $(0.5 \%)$, and hydrocephalus $(0.5 \%)$. ICA injury occurred in seven patients $(1.8 \%)$, and two of them were treated with endovascular treatment [39]. Six patients (1.6\%) died of complications. The complications were also compared between EMA and MLOA (Supplementary Table 3 ).

To be mentioned, two patients have CSF leakage after RT. One of them was treated by lumbar drainage, and another one was treated by transnasal endoscopic repair surgery.

\section{Effects of RT on long-term outcomes when GTR was achieved}

A total of 89 patients with primary SBCs achieved GTR. PFS was significantly longer in the group with pre-recurrence $\mathrm{RT}$ than in the others $(\mathrm{HR}=3.41,95 \% \mathrm{CI}=1.39-8.35$, $P=0.007$ ) after adjusting for age, sex, and tumor volume. CSS was not evaluated due to the limited number of death events.

Similarly, we analyzed the effect of RT in 95 patients with primary SBCs who did not achieve GTR. Compared with no pre-recurrence RT, pre-recurrence RT also showed significant positive effect on PFS ( $\mathrm{HR}=2.50,95 \% \mathrm{CI}=1.30-4.84$, $P=0.006)$. 
Table 4 Risk factors associated with failure of gross-total resection. Analysis based on multivariable logistic regression

\begin{tabular}{|c|c|c|c|c|}
\hline \multicolumn{2}{|l|}{ Variable } & \multirow{2}{*}{$\frac{\mathrm{OR}}{1.49}$} & \multirow{2}{*}{$\begin{array}{l}95 \% \text { CI } \\
0.78-2.85\end{array}$} & \multirow{2}{*}{$\frac{P}{.223}$} \\
\hline Sex & Female vs. male & & & \\
\hline \multirow[t]{2}{*}{$\operatorname{Age}^{\mathrm{a}}$} & Group 2 vs. group 1 & 0.28 & $0.09-0.87$ & .028 \\
\hline & Group 3 vs. group 1 & 0.27 & $0.07-1.02$ & .054 \\
\hline Histopathologic subtype & $\begin{array}{l}\text { Dedifferentiated vs. conven- } \\
\text { tional/chondroid }\end{array}$ & 1.35 & $0.09-20.19$ & .826 \\
\hline History of surgery & Yes vs. no & 3.45 & $1.59-7.49$ & .002 \\
\hline History of RT & Yes vs. no & 0.80 & $0.27-2.34$ & .686 \\
\hline \multirow[t]{3}{*}{ Tumor location (sagittal view) } & Lower $2 / 3$ vs. upper $2 / 3$ & 8.73 & $3.12-24.45$ & $<.001$ \\
\hline & Total clivus vs. upper $2 / 3$ & 3.07 & $1.36-6.94$ & .007 \\
\hline & Others vs. upper $2 / 3$ & 1.66 & $0.30-9.16$ & .561 \\
\hline Tumor location (axial view) ${ }^{a}$ & Paramedian vs. midline & 2.88 & $1.42-5.82$ & .003 \\
\hline Cavernous sinus invasion & Yes vs. no & 1.72 & $0.81-3.67$ & .158 \\
\hline Dural penetration & Yes vs. no & 1.73 & $0.90-3.32$ & .101 \\
\hline Tumor volume & $>40$ vs. $\leq 40 \mathrm{~cm}^{3}$ & 3.07 & $1.22-7.76$ & .018 \\
\hline
\end{tabular}

${ }^{a}$ Age was classified into three groups, e.g., group 1 represented $\leq 20$ years of age, group 2 represented between 20 and 60 years of age, and group $C$ represented $\geq 60$ years of age. ${ }^{\mathrm{b}}$ One patient whose tumor was located in the paramedian region was included in the group with median extension to the paramedian region

The bolded entries are significant $p$-values

\section{Radiotherapy modalities and outcomes}

To study the relationship between RT modalities and outcomes, we analyzed 45 patients with primary SBCs who were treated with either CPRT or other RT modalities. However, because of the limited statistical power due to the short follow-up and limited number of cases in the group with CPRT, we did not observe significant differences in CSS or PFS between the two groups (Supplementary Table 4).

\section{Discussion}

\section{Choice of surgical approach}

In present series, both EMA and MLOA were used to resect SBCs. We did not find a significant difference in the GTR rate between EMA and MLOA, which was consistent with the previous findings of our group and others [30, 34, 37]. To be mentioned, most of our cases were resected through EMAs, which is mainly determined by tumor's origin sites and extending directions $[10,23,26]$. EMA has advantage in reducing injury of cranial nerve compared with MLOA, because the tumor can be safely resected without crossing the nerves in most circumstances [17]. However, the most serious and urgent complication is ICA injury. A strategy for expanding exposure for ICA must be planed carefully according to the tumor extending direction pre-operatively; meanwhile, an endovascular treatment should be available just in case for this rare complication [39]. Although seven cases $(2.0 \%)$ experienced ICA injury in present series, they were recovered well except one patient with recurrent chordoma died of ICA occlusion in our early stage. In summary, we regard that EEA is the first choice for SBCs, and it is also safe when the surgeons have enough experience [7]. A neurosurgeon who majored in the treatment of SBCs should master both EMA and MLOA [1].

\section{Resection rate and outcome}

The resection rate strongly influences CSS and PFS $[8,37$, 38]. The maximum resection was traditionally recommended with the goal of marginal resection $[24,25,30]$. The recurrence rate ranges from 16 to $45 \%$ at 10 years after marginal resection, and most recurrences occur within 2 or 3 years $[14,23,34]$. After a more detailed analysis, we found that the advantage of marginal resection was mainly contributed by GTR and that no obvious differences were found among NTR, STR, and PR. Therefore, GTR should be the goal if possible [23]. This result also indicates that the standard for assessing the resection rate should be postsurgical images rather than the intraoperative impression of the surgeon [6].

\section{Other risk factors for outcome}

Dedifferentiated subtype, history of RT, lack of postsurgical $\mathrm{RT}$, and metastasis increased the risk of both shorter survival and recurrence, which has been shown by previous studies [5, $8,10,17,34]$. However, it is controversial whether different survival rates exist between conventional and chondroid types $[7,29]$. We found no significant difference, which is consistent with several studies [2, 32]. Several previous studies showed 
that dural penetration $[7,36]$ and age $[5,29]$ were associated with long-term outcomes, and we demonstrated that they were not independent risk factors after multivariate analysis [23,38].

\section{Risk factors reducing the chances of GTR}

We found that younger age ( $\leq 20$ years) increased the risk of non-GTR. This may be explained by our previous finding that most children with SBCs had these tumors in the lower clivus and craniocervical junction area [3]. Tumors in the lower clivus have the lowest GTR rate [3, 6, 14, 17]. Not surprisingly, history of surgery, large tumor volume, and tumor location are independent risk factors for GTR, as supported by previous studies $[7,14,17,26,30]$.

\section{Surgical complications and treatments}

CSF leakage and cranial nerve injury were the main complications in prior studies $[6,14,17]$. Benefiting mainly from the application of vascularized nasoseptal flaps [7, 17], CSF leakage was low in our group (3.9\%). We emphasize the importance of cleaning tumors that are attached to the dura while maintaining the integrity of the dura, which effectively prevent CSF leakage. Radical resection of all involved dura might account for high rates of CSF leakage (14\%) [6, 9]. We deem the dura to be a natural barrier against tumor growth intracranially, which has been supported indirectly by two autopsy studies [21, 28]. Both studies found that the dura was intact even in advanced stages of SBCs.

Chordomas with cavernous sinus invasion have a lower GTR rate (23.0\% vs $47.7 \%$ ), although it is not an independent risk factor. The major difficulty for achieving GTR in this setting was fear of ICA injury, which is another serious complication [17]. Endovascular treatment is an effective remedial treatment when the ICA is injured [39]. Therefore, we support the notion that EMA is safe and has few complications once adequate experience is gained [26].

\section{Timing of RT and outcome}

Adjuvant RT is a widely accepted treatment for residual chordomas [19, 40], although no strong evidence supports it [31]. We found that PFS and CSS were significantly longer in patients with pre-recurrence RT than in other patients, which is similar to prior studies [14, 34]. In addition, the PFS in the late RT group was shortest in the present series (data not shown); however, the CSS was still longer than that of the patients who had no RT. Similarly, Olabisi Sanusi et al. found that a second dose of RT at any point either as sole treatment or as adjuvant treatment for recurrence showed a statistically significant effect on PFS $(\mathrm{P}=0.009)$ [22]. Taken together, these findings indicate that adjuvant RT is an independent factor for better outcomes [7, 34], and the importance of pre-recurrence RT is worth emphasizing. Once recurrence occurs, postsurgical RT is also recommended to prolong CSS when the patient has no RT history [25].

\section{RT after GTR}

As expected, our finding of pre-recurrence RT in patients who failed GTR strengthens the positive role of RT in postponing recurrence $[7,22]$. However, it remains debatable whether to undertake pre-recurrence RT after GTR $[8,19$, 23]. Lately, Yagiz Yolcu et al.'s found that compared with GTR alone, GTR plus RT did not offer any significant survival benefit for patients with sacral or spinal chordomas, at the price of higher complications rate [33]. However, our result is consistent with a recent finding that pre-recurrence RT in GTR group will prolong PFS [22]. Therefore, prerecurrence $\mathrm{RT}$ is recommended to prevent recurrence even after GTR for patients with SBCs [19].

\section{Radiation modalities and outcome}

In recent years, increasing studies found that CPRT has advantages over other modalities in the chordoma treatment. Satoshi Takahashi et al. [27] found that the PFS of carbon ion treated group was longer than that of the other groups treated with other radiation modalities or untreated; however, a recent meta-analysis did not find significant differences between radiosurgery, proton RT, and carbon ion $\mathrm{RT}$ at 3 and 5 years survival [40]. Li $\mathrm{H}$ et al. also found that IMRT serves as an effective alternative to CPRT based on their retrospective analysis of 46 cases. Selection bias was likely present in Li's study and might be conducive to a result that contradicts previous findings; for example, economic status (CPRT is much more expensive than other RT modalities) and education status (which will affect whether the patients would follow the clinical recommendation) influence the accessibility of CPRT. For now, we cannot compare the effectiveness of CPRT and other radiation modalities due to short follow-up and limited number of cases with CPRT. Thus, we cannot rule out the possibility that there is no benefit of CPRT over other types of radiation, which might be associated with the improved RT techniques of radiosurgery or IMRT $[15,16]$. Although we recommend that patients with chordomas seek help from radiologists who administer particle radiation therapy, we firmly believe that a randomized trial is warranted even in its rarity [14].

\section{Limitations}

As a retrospective study, our study has multiple limitations, including the lack of well-designed timing of RT and random choice of radiation modalities. In addition, we cannot assess whether pre-recurrence RT improves CCS in patients 
who have achieved GTR $(n=114)$ due to the limited number of death events $(n=14)$ in the current study. We will continue following up in order to evaluate the effect of prerecurrence RT. Moreover, tumor biological characteristics were not analyzed; these characteristics might be underlying factors for GTR [8]. Recently, we found that genomic alterations were associated with recurrence and CSS [4]. Therefore, combining tumor biology with clinical characteristics will produce more robust conclusions in the future.

\section{Conclusions}

To the best of our knowledge, this is the largest series of SBCs surgically treated by single surgical team, and we found that surgery should be the initial treatment for primary SBCs. GTR is the surgical goal and should be applied in both primary and recurrent SBCs [38]. History of surgery, larger tumor volume, and tumor location (lower clivus, extension from the midline to the paramedian region) are independent risk factors for GTR. Pre-recurrence RT will postpone recurrence, even after GTR is achieved.

Supplementary Information The online version contains supplementary material available at https://doi.org/10.1007/s10143-021-01576-4.

Acknowledgements The authors thank Xiaohong R. Yang for editing assistance. The authors thank Xixi Feng, who assisted in the illustration for Fig. 1.

Author contributions Jiwei Bai: Conceptualization, methodology, formal analysis, writing —original draft, and writing — review and editing.

Mingxuan Li: Methodology, acquisition of data, validation, and writing-review.

Jianxin Shi: Formal analysis.

Liwei Jing: Formal analysis.

Yixuan Zhai: Methodology, acquisition of data, and validation.

Shuheng Zhang: Methodology and acquisition of data.

Junmei Wang: Methodology.

Peng Zhao: Acquisition of data.

Chuzhong Li: Acquisition of data.

Songbai Gui: Acquisition of data and supervision.

Yazhuo Zhang: Conceptualization, supervision, and project administration.

Funding This research was supported by Beijing Municipal Science and Technology Commission (Z171100000117002, Yazhuo Zhang), Research Special Fund for Public Welfare Industry of Health (201402008, Yazhuo Zhang).

Availability of data and material Not applicable.

Code availability Not applicable.

\section{Declarations}

Ethics approval This retrospective study was approved by the ethics committee of Beijing Tiantan Hospital.
Consent to participate All patients or their relatives gave their informed consent within written treatment contract on admission and therefore prior to their inclusion in the study.

Consent for publication Not applicable.

Conflict of interest The authors declare no competing interests.

Open Access This article is licensed under a Creative Commons Attribution 4.0 International License, which permits use, sharing, adaptation, distribution and reproduction in any medium or format, as long as you give appropriate credit to the original author(s) and the source, provide a link to the Creative Commons licence, and indicate if changes were made. The images or other third party material in this article are included in the article's Creative Commons licence, unless indicated otherwise in a credit line to the material. If material is not included in the article's Creative Commons licence and your intended use is not permitted by statutory regulation or exceeds the permitted use, you will need to obtain permission directly from the copyright holder. To view a copy of this licence, visit http://creativecommons.org/licenses/by/4.0/.

\section{References}

1. Al-Mefty O (2017) Chordoma. Acta Neurochir 159:1869-1871. https://doi.org/10.1007/s00701-017-3287-9

2. Almefty K, Pravdenkova S, Colli BO, Al-Mefty O, Gokden M (2007) Chordoma and chondrosarcoma: similar, but quite different, skull base tumors. Cancer 110:2457-2467. https://doi.org/10. 1002/cncr.23073

3. Bai J, Zhang S, Zhai Y, Wang S, Li M, Li C, Zhao P, Geng S, Gui S, Zhang Y (2020) A series of 62 skull base chordomas in pediatric and adolescent patients: clinical characteristics, treatments, and outcomes. Neurol India 68:1030-1036. https://doi.org/ 10.4103/0028-3886.294548

4. Bai J, Shi J, Li C, Wang S, Zhang T, Hua X, Zhu B, Koka H, Wu HH, Song L, Wang D, Wang M, Zhou W, Ballew BJ, Zhu B, Hicks B, Mirabello L, Parry DM, Zhai Y, Li M, Du J, Wang J, Zhang S, Liu Q, Zhao P, Gui S, Goldstein AM, Zhang Y, Yang XR (2021) Whole genome sequencing of skull-base chordoma reveals genomic alterations associated with recurrence and chordomaspecific survival. Nat Commun 12:757. https://doi.org/10.1038/ s41467-021-21026-5

5. Beccaria K, Tauziede-Espariat A, Monnien F, Adle-Biassette H, Masliah-Planchon J, Pierron G, Maillot L, Polivka M, Laquerriere A, Bouillot-Eimer S, Gimbert E, Gauchotte G, Coffinet L, Sevestre H, Alapetite C, Bolle S, Thompson D, Bouazza S, George B, Zerah M, Sainte-Rose C, Puget S, Varlet P (2018) Pediatric chordomas: results of a multicentric study of 40 children and proposal for a histopathological prognostic grading system and new therapeutic strategies. J Neuropathol Exp Neurol 77:207-215. https:// doi.org/10.1093/jnen/nlx118

6. Brito da Silva H, Straus D, Barber JK, Rostomily RC, Ferreira M Jr, Sekhar LN (2018) Cranial chordoma: a new preoperative grading system. Neurosurgery 83:403-415. https://doi.org/10.1093/ neuros/nyx 423

7. Cavallo LM, Mazzatenta D, d'Avella E, Catapano D, Fontanella MM, Locatelli D, Luglietto D, Milani D, Solari D, Vindigni M, Zenga F, Zona G, Cappabianca P (2020) The management of clival chordomas: an Italian multicentric study. J Neurosurg 1-10. https://doi.org/10.3171/2020.5.JNS20925

8. Di Maio S, Temkin N, Ramanathan D, Sekhar LN (2011) Current comprehensive management of cranial base chordomas: 10 -year 
meta-analysis of observational studies. J Neurosurg 115:10941105. https://doi.org/10.3171/2011.7.JNS11355

9. Di Maio S, Rostomily R, Sekhar LN (2012) Current surgical outcomes for cranial base chordomas: cohort study of 95 patients. Neurosurgery 70:1355-1360. discussion 1360. https://doi.org/10. 1227/NEU.0b013e3182446783

10. Fernandez-Miranda JC, Gardner PA, Snyderman CH, Devaney KO, Mendenhall WM, Suarez C, Rinaldo A, Ferlito A (2014) Clival chordomas: a pathological, surgical, and radiotherapeutic review. Head Neck 36:892-906. https://doi.org/10.1002/hed. 23415

11. Frezza AM, Botta L, Trama A, Dei Tos AP, Stacchiotti S (2019) Chordoma: update on disease, epidemiology, biology and medical therapies. Curr Opin Oncol 31:114-120. https://doi.org/10.1097/ CCO.0000000000000502

12. Gui S, Zong X, Wang X, Li C, Zhao P, Cao L, Zhang Y (2016) Classification and surgical approaches for transnasal endoscopic skull base chordoma resection: a 6-year experience with 161 cases. Neurosurg Rev 39:321-332. discussion 332-323. https:// doi.org/10.1007/s10143-015-0696-1

13. Hung GY, Horng JL, Yen HJ, Yen CC, Chen WM, Chen PC, Wu HT, Chiou HJ (2014) Incidence patterns of primary bone cancer in Taiwan (2003-2010): a population-based study. Ann Surg Oncol 21:2490-2498. https://doi.org/10.1245/s10434-014-3697-3

14. Jahangiri A, Chin AT, Wagner JR, Kunwar S, Ames C, Chou D, Barani I, Parsa AT, McDermott MW, Benet A, El-Sayed IH, Aghi MK (2015) Factors predicting recurrence after resection of clival chordoma using variable surgical approaches and radiation modalities. Neurosurgery 76:179-185; discussion 185-176. https:// doi.org/10.1227/NEU.0000000000000611

15. Kano H, Iqbal FO, Sheehan J, Mathieu D, Seymour ZA, Niranjan A, Flickinger JC, Kondziolka D, Pollock BE, Rosseau G, Sneed PK, McDermott MW, Lunsford LD (2011) Stereotactic radiosurgery for chordoma: a report from the North American Gamma Knife Consortium. Neurosurgery 68:379-389. https://doi.org/10. 1227/NEU.0b013e3181ffa12c

16. Kim JW, Suh CO, Hong CK, Kim EH, Lee IJ, Cho J, Lee KS (2017) Maximum surgical resection and adjuvant intensity-modulated radiotherapy with simultaneous integrated boost for skull base chordoma. Acta Neurochir 159:1825-1834. https://doi.org/ 10.1007/s00701-016-2909-y

17. Koutourousiou M, Gardner PA, Tormenti MJ, Henry SL, Stefko ST, Kassam AB, Fernandez-Miranda JC, Snyderman CH (2012) Endoscopic endonasal approach for resection of cranial base chordomas: outcomes and learning curve. Neurosurgery 71:614-624. discussion 624-615. https://doi.org/10.1227/NEU.0b013e3182 $5 \mathrm{ea} 3 \mathrm{e} 0$

18. Li C, Zhu H, Zong X, Wang X, Gui S, Zhao P, Zhang Y (2018) History, current situation, and future development of endoscopic neurosurgery in China. World Neurosurg 110:270-275. https:// doi.org/10.1016/j.wneu.2017.11.103

19. Li H, Zhang H, Hu L, Wang H, Wang D (2020) Endoscopic endonasal resection and radiotherapy as treatment for skull base chordomas. Acta Otolaryngol 140:789-794. https://doi.org/10.1080/ 00016489.2020 .1748225

20. McMaster ML, Goldstein AM, Bromley CM, Ishibe N, Parry DM (2001) Chordoma: incidence and survival patterns in the United States, 1973-1995. Cancer Causes Control 12:1-11. https://doi. org/10.1023/a:1008947301735

21. Oikawa S, Kyoshima K, Goto T, Iwashita T, Takizawa T, Kobayashi S, Ito M (2001) Histological study on local invasiveness of clival chordoma. Case report of autopsy Acta Neurochir (Wien) 143:1065-1069. https://doi.org/10.1007/s007010170013

22. Sanusi O, Arnaout O, Rahme RJ, Horbinski C, Chandler JP (2018) Surgical resection and adjuvant radiation therapy in the treatment of skull base chordomas. World neurosurgery 115:e13-e21. https://doi.org/10.1016/j.wneu.2018.02.127

23. Sen C, Triana AI, Berglind N, Godbold J, Shrivastava RK (2010) Clival chordomas: clinical management, results, and complications in 71 patients. J Neurosurg 113:1059-1071. https://doi.org/ 10.3171/2009.9.JNS08596

24. Stacchiotti S, Sommer J, Chordoma Global Consensus G (2015) Building a global consensus approach to chordoma: a position paper from the medical and patient community. Lancet Oncol 16:e71-83. https://doi.org/10.1016/S1470-2045(14)71190-8

25. Stacchiotti S, Gronchi A, Fossati P, Akiyama T, Alapetite C, Baumann M, Blay JY, Bolle S, Boriani S, Bruzzi P, Capanna R, Caraceni A, Casadei R, Colia V, Debus J, Delaney T, Desai A, Dileo P, Dijkstra S, Doglietto F, Flanagan A, Froelich S, Gardner PA, Gelderblom H, Gokaslan ZL, Haas R, Heery C, Hindi N, Hohenberger P, Hornicek F, Imai R, Jeys L, Jones RL, Kasper B, Kawai A, Krengli M, Leithner A, Logowska I, Martin Broto J, Mazzatenta D, Morosi C, Nicolai P, Norum OJ, Patel S, Penel N, Picci P, Pilotti S, Radaelli S, Ricchini F, Rutkowski P, Scheipl S, Sen C, Tamborini E, Thornton KA, Timmermann B, Torri V, Tunn PU, Uhl M, Yamada Y, Weber DC, Vanel D, Varga PP, Vleggeert-Lankamp CLA, Casali PG, Sommer J (2017) Best practices for the management of local-regional recurrent chordoma: a position paper by the Chordoma Global Consensus Group. Ann Oncol 28:1230-1242. https://doi.org/10.1093/annonc/mdx054

26. Stippler M, Gardner PA, Snyderman CH, Carrau RL, Prevedello DM, Kassam AB (2009) Endoscopic endonasal approach for clival chordomas. Neurosurgery 64:268-277. discussion 277-268. https://doi.org/10.1227/01.NEU.0000338071.01241.E2

27. Takahashi S, Kawase T, Yoshida K, Hasegawa A, Mizoe JE (2009) Skull base chordomas: efficacy of surgery followed by carbon ion radiotherapy. Acta Neurochir 151:759-769. https://doi.org/ 10.1007/s00701-009-0383-5

28. Tamaki M, Aoyagi M, Kuroiwa T, Yamamoto M, Kishimoto S, Ohno K (2007) Clinical course and autopsy findings of a patient with clival chordoma who underwent multiple surgeries and radiation during a 10-year period. Skull Base 17:331-340. https://doi. org/10.1055/s-2007-986438

29. Tian K, Wang L, Wang K, Ma J, Li D, Hao S, Yang Y, Du J, Jia G, Zhang L, Wu Z, Zhang J (2016) Analysis of clinical features and outcomes of skull base chordoma in different age-groups. World neurosurgery 92:407-417. https://doi.org/10.1016/j.wneu.2016. 05.035

30. Wang L, Wu Z, Tian K, Wang K, Li D, Ma J, Jia G, Zhang L, Zhang J (2017) Clinical features and surgical outcomes of patients with skull base chordoma: a retrospective analysis of 238 patients. J Neurosurg 127:1257-1267. https://doi.org/10.3171/2016.9. JNS16559

31. Wright CH, Wright J, Cioffi G, Hdeib A, Kasliwal MK, Kruchko C, Barnholtz-Sloan JS, Sloan AE (2019) Association of cancer center type with treatment patterns and overall survival for patients with sacral and spinal chordomas: an analysis of the National Cancer Database from 2004 to 2015. J Neurosurg Spine 1-10. https://doi.org/10.3171/2019.7.SPINE19566

32. Yasuda M, Bresson D, Chibbaro S, Cornelius JF, Polivka M, Feuvret L, Takayasu M, George B (2012) Chordomas of the skull base and cervical spine: clinical outcomes associated with a multimodal surgical resection combined with proton-beam radiation in 40 patients. Neurosurg Rev 35:171-182. discussion 182-173. https://doi.org/10.1007/s10143-011-0334-5

33. Yolcu Y, Wahood W, Alvi MA, Kerezoudis P, Okuno SH, Foote RL, Bydon M (2019) Evaluating the role of adjuvant radiotherapy in the management of sacral and vertebral chordoma: results from a national database. World Neurosurgery 127:e1137-e1144. https://doi.org/10.1016/j.wneu.2019.04.070 
34. Yumiko O, Tamura R, Takahashi S, Morimoto Y, Sato M, Horikoshi T, Hassaan S, Yoshida K, Toda M (2020) A comparative study between traditional microscopic surgeries and endoscopic endonasal surgery for skull base chordomas. World Neurosurgery 134:e1099-e1107. https://doi.org/10.1016/j.wneu.2019.11.113

35. Zenonos GA, Fernandez-Miranda JC, Mukherjee D, Chang YF, Panayidou K, Snyderman CH, Wang EW, Seethala RR, Gardner PA (2018) Prospective validation of a molecular prognostication panel for clival chordoma. J Neurosurg 1:1-10. https://doi.org/10. 3171/2018.3.JNS172321

36. Zhai Y, Bai J, Wang S, Du J, Wang J, Li C, Gui S, Zhang Y (2017) Differences in dural penetration of clival chordomas are associated with different prognosis and expression of platelet-derived growth factor receptor-beta. World neurosurgery 98:288-295. https://doi. org/10.1016/j.wneu.2016.07.096

37. Zhai Y, Bai J, Wang S, Gao H, Li M, Li C, Gui S, Zhang Y (2018) Analysis of clinical factors and PDGFR-beta in predicting prognosis of patients with clival chordoma. J Neurosurg 129:1429-1437. https://doi.org/10.3171/2017.6.JNS17562
38. Zhai Y, Bai J, Li M, Wang S, Li C, Wei X, Zhang Y (2019) A nomogram to predict the progression-free survival of clival chordoma. J Neurosurg 1:1-9. https://doi.org/10.3171/2019.10.JNS19 2414

39. Zhang Y, Tian Z, Li C, Liu J, Zhang Y, Yang X, Zhang Y (2019) A modified endovascular treatment protocol for iatrogenic internal carotid artery injuries following endoscopic endonasal surgery. J Neurosurg 1:1-8. https://doi.org/10.3171/ 2018.8.JNS181048

40. Zhou J, Yang B, Wang X, Jing Z (2018) Comparison of the effectiveness of radiotherapy with photons and particles for chordoma after surgery: a meta-analysis. World neurosurgery 117:46-53. https://doi.org/10.1016/j.wneu.2018.05.209

Publisher's note Springer Nature remains neutral with regard to jurisdictional claims in published maps and institutional affiliations. 